\title{
COMPOSIČ̃̃ E CINÉTICA DE DEGRADAČ̃̃O DO ÁCIDO ASCÓRBICO EM RAÇÕES PARA AQUICULTURA
}

\author{
Composition and degradation kinetics of ascorbic acid in rations for aquiculture
}

\author{
Ricardo de Figueiredo Guilherme1, José Marcelino Oliveira Cavalheiro², Olivaldo Lacerda Brasileiro³, \\ João Paulo de Sousa Prado ${ }^{4}$, Thiago Brandão Cavalheiro 5
}

\begin{abstract}
RESUMO
O crescente desenvolvimento da aquicultura nos últimos anos vem despertando o interesse de se estudar a melhor forma de utilização das rações, visto que estas podem elevar em até $60 \%$ o custo total de produção. O nível de inclusão de ácido ascórbico em uma dieta deve considerar a variação da matéria-prima, as interações entre nutrientes e as perdas no processamento, estocagem e lixiviação. $\mathrm{O}$ estudo foi conduzido para avaliar a estabilidade térmica, fotoquímica e lixiviação do ácido ascórbico em diferentes rações e no premix vitamínico utilizado na aquicultura. As amostras 1 (Ração peletizada e posteriormente desintegrada, destinada à alimentação de camarões marinhos pós-larvas $\left(\mathrm{PL}^{2}-\mathrm{PL}^{15}\right.$ ) e 5 (premix vitamínico) foram as que apresentaram as maiores perdas de ácido ascórbico durante a degradação térmica, fotoquímica e lixiviação.
\end{abstract}

Termos para indexação: Nutrição animal, ácido ascórbico, estabilidade, estocagem.

\section{ABSTRACT}

The increasing development of the aquiculture in the last years has stimulated the interest in studying the best form of using the rations, because they increase in up to $60 \%$ the total cost of the production. The level of ascorbic acid incorporated in a diet must consider the variation of the raw material, the interactions between nutrients and the losses in the processing, storage, and leaching. The study was conducted to evaluate the thermal stability, photochemistry, and leaching of ascorbic acid on different rations and vitamin premix used in the aquiculture. Samples 1 (pelletized and later disintegrated ration, destined to feeding after-larvae marine shrimps (PL2-PL15) and 5 (vitamin premix) presented the biggest ascorbic acid losses during the thermal degradation, photochemistry, and leaching.

Index terms: Animal nutrition, ascorbic acid, stability.

(Recebido em 22 de abril de 2008 e aprovado em 25 de março de 2009)

\section{INTRODUÇÃO}

A aquicultura atualmente constitui-se em um dos sistemas de produção que mais cresce no mundo. Em seu crescente desenvolvimento nos últimos anos, vem utilizando diferentes espécies de animais aquáticos na tentativa de atender a uma demanda global por alimento que a cada dia se acentua (Almeida et al., 2004). Os crustáceos vêm se destacando pelo seu valor nutritivo e por sua enorme capacidade de adaptação às mais variadas condições de cultivo (Ormond et al., 2004). As vitaminas são compostos nutricionais fundamentais para os organismos, sendo que muitos processos fisiológicos e bioquímicos dependem dos suprimentos adequados das mesmas, tendo essencial importância na nutrição de peixes e camarões.
O ácido ascórbico é um micronutriente essencial na dieta de camarão, podendo sofrer degradação por vários fatores como: tempo, temperatura, luminosidade, oxigênio, pH e lixiviação (Fish Farming International-FFI, 1984). Esse ácido é considerado extremamente lábil em seu aspecto químico, e acredita-se que uma das causas da sua degradação seja a radiação luminosa (Soliman et al., 1987).

O ácido L-ascórbico na sua forma cristalina, seca e pura é razoavelmente estável. Entretanto, é facilmente oxidável em condições neutras e alcalinas, quando o oxigênio, a umidade, os microelementos, as temperaturas elevadas, a luz e os lipídios oxidados promovem sua oxidação e destruição (O'Keefe, 2001). Por essas razões, perdas dessa vitamina podem ocorrer durante a industrialização e o prolongado armazenamento das rações

\footnotetext{
${ }^{1}$ Médico Veterinário, Mestre em Ciência e Tecnologia de Alimentos- Campus I - Universidade Federal da Paraíba/UFPB - Rua Franca Filho, 538 - João Pessoa, PB. e-mail: ricardo.figueiredo@hotmail.com

${ }^{2}$ Professor, Doutor no Programa de Pós-Graduação em Ciência e Tecnologia de Alimentos - Campus I Universidade Federal da Paraíba/UFPB - 58059900 - João Pessoa, PB - jmarcelin@uol.com.br

${ }^{3}$ Farmacêutico-Bioquímico, Mestre em Ciência e Tecnologia de Alimentos, Pesquisador pelo CNPq - UFPB - Rua João Junqueira Viana, 13 - João Pessoa, PB - olivaldolacerda@hotmail.com

${ }^{4}$ Químico, Mestrando em Ciência e Tecnologia de Alimentos - Campus I - Universidade Federal da Paraíba/UFPB - Rua Antonio Leopoldo Batista, 105 CEP: 58051-110 - João Pessoa, PB -jp_prado@hotmail.com

${ }^{5}$ Graduando em Engenharia de Alimentos- Campus I - Universidade Federal da Paraíba/UFPB - 58059-900 - João Pessoa, PB- thiagobcav@hotmail.com
} 
(Tacon, 1991). Cerca de 20\% do ácido ascórbico adicionado às rações para aquicultura são degradados durante o processamento e armazenagem da mesma (Eva et al., 1976).

As rações para a fase pós-larva de camarão são de textura muito fina (geralmente menor que $0,5 \mathrm{~mm}$ ) e, assim, estão sujeitas a excessivas perdas de nutrientes por dissolução ou lixiviação. Assim, rações devem apresentar adequada flutuabilidade na água, reduzindo a sua superfície de contato e, portanto, reduzindo as perdas de nutrientes por lixiviação (Kubitza, 1999).

Conduziu-se este trabalho, com o objetivo de avaliar a estabilidade térmica, fotoquímica e lixiviatória do ácido ascórbico em diferentes rações comerciais e em premix vitamínico utilizados na aquicultura.

\section{MATERIAL E MÉTODOS}

As análises foram realizadas utilizando-se quatro rações comerciais e um premix vitamínico, com as seguintes características: 1)Ração peletizada e, posteriormente, desintegrada, destinada à alimentação de camarões marinhos pós-larvas ( $\left.\mathrm{PL}^{2}-\mathrm{PL}^{15}\right)$. 2) Ração peletizada e, posteriormente, desintegrada, destinada à alimentação de camarões marinhos na fase de pós-larvas ( $\mathrm{PL}^{7}-\mathrm{PL}^{25} .3$ ) Ração peletizada e, posteriormente, desintegrada, destinada à alimentação de camarões marinhos com peso médio entre 1 e 3 gramas. 4) Ração peletizada destinada à alimentação de camarões marinhos desde a fase juvenil (com peso médio de 3,0 gramas) até atingir peso de mercado. 5)Premix vitamínico sob a forma de monofosfato de ácido ascórbico (Lutavit ${ }^{\mathrm{R}} \mathrm{C}$ monophosphate Feed Grade).

$\mathrm{Na}$ análise de degradação térmica, as amostras foram armazenadas sob as seguintes condições: geladeira $\left(6^{\circ} \mathrm{C}\right.$ $\left.\pm 2{ }^{\circ} \mathrm{C}\right)$, ambiente climatizado $\left(20^{\circ} \mathrm{C} \pm 3{ }^{\circ} \mathrm{C}\right)$, e em estufa $(40$ ${ }^{\circ} \mathrm{C} \pm 3{ }^{\circ} \mathrm{C}$ ). As rações foram acondicionadas em sacos confeccionados com a embalagem original (ráfia) e, posteriormente, colocados nestes 03 (três) ambientes. $\mathrm{O}$ teor de ácido ascórbico foi determinado nos seguintes períodos: $05,10,15,20,25$ e 30 dias, nas condições anteriormente descritas.

Para avaliação da estabilidade fotoquímica do ácido ascórbico, as amostras foram armazenadas sob as seguintes condições: $10 \mathrm{~g}$ de cada amostra foram colocadas em uma estufa desligada (escuro) e em uma estufa com exposição à luz (lâmpada de 60 watts) e analisadas em triplicatas no intervalo de 04, 08 e 12 dias.

Para a verificação da degradação por lixiviação, apenas as rações foram submetidas ao seguinte processo: as amostras foram colocadas em contato com água salgada (marinha) e doce (rio Paraíba) e analisadas em diferentes intervalos de tempo (3, 6 e 12 horas). A determinação de ácido ascórbico foi realizada utilizando-se o método padrão da AOAC (1984), modificado por Benassi \& Antunes (1988). As determinações foram realizadas em triplicata.

\section{RESULTADOS E DISCUSSÃO}

As amostras foram acondicionadas em vários ambientes: em geladeira, temperatura ambiente e estufa para avaliar a estabilidade. A temperatura de $40^{\circ} \mathrm{C}$ é a mesma encontrada em vários locais de armazenamento dessas rações como caminhões de transporte e até nos próprios criatórios de camarão que, inclusive, em sua maioria, são localizados na região Nordeste.

No estudo de análise luminosa as amostras avaliadas foram mantidas à temperatura ambiente com sistema de iluminação que não oferecia energia térmica suficiente para alterar a temperatura das amostras.

$\mathrm{Na}$ amostra 01 , ocorreram as maiores perdas de ácido ascórbico em relação às outras amostras, pois essa, inicialmente, apresentou $300 \mathrm{mg} / \mathrm{Kg}$ e no $30^{\circ}$ dia submetida à temperatura de geladeira perdeu $46,76 \%$, no ambiente climatizado perdeu $47,33 \%$ e na estufa chegou a perder $50,67 \%$, de acordo com as tabelas 1, 2 e 3 .

Conforme as tabelas 1, 2 e 3, as amostras 02 e 03 ao serem analisadas apresentaram $150 \mathrm{mg} / \mathrm{Kg}$ de ácido

Tabela 1 - Degradação de ácido ascórbico em rações e premix expostos a ambiente de geladeira $\left(6^{\circ} \mathrm{C} \pm 2^{\circ} \mathrm{C}\right)$.

\begin{tabular}{cccccc}
\hline $\begin{array}{c}\text { Período de degradação } \\
\% \text { (dias) }\end{array}$ & Amostra 1 & Amostra 2 & Amostra 3 & Amostra 4 & Amostra 5 \\
\hline T5 & $8,30^{\mathrm{f}} \pm 0,13$ & $13,49^{\mathrm{c}} \pm 3,61$ & $10,66^{\mathrm{f}} \pm 0,40$ & $0,34^{\mathrm{c}} \pm 0,55$ & $2,27^{\mathrm{f}} \pm 0,03$ \\
T10 & $34,67^{\mathrm{e}} \pm 0,45$ & $12,66^{\mathrm{c}} \pm 0,10$ & $11,33^{\mathrm{e}} \pm 0,48$ & $0,78^{\mathrm{bc}} \pm 0,80$ & $2,62^{\mathrm{e}} \pm 0,00$ \\
T15 & $41,55^{\mathrm{d}} \pm 0,20$ & $14,11^{\mathrm{c}} \pm 0,16$ & $12,00^{\mathrm{d}} \pm 0,09$ & $1,48^{\mathrm{b}} \pm 0,44$ & $4,37^{\mathrm{d}} \pm 0,01$ \\
T20 & $42,66^{\mathrm{c}} \pm 0,11$ & $16,66^{\mathrm{b}} \pm 0,21$ & $12,66^{\mathrm{c}} \pm 0,28$ & $1,49^{\mathrm{b}} \pm 0,64$ & $9,87^{\mathrm{c}} \pm 0,00$ \\
T25 & $44,67^{\mathrm{b}} \pm 0,39$ & $18,22^{\mathrm{ab}} \pm 0,79$ & $13,33^{\mathrm{b}} \pm 0,39$ & $2,96^{\mathrm{a}} \pm 0,36$ & $10,00^{\mathrm{b}} \pm 0,00$ \\
T30 & $46,76^{\mathrm{a}} \pm 0,09$ & $20,00^{\mathrm{a}} \pm 0,46$ & $14,00^{\mathrm{a}} \pm 0,11$ & $3,70^{\mathrm{a}} \pm 0,24$ & $10,87^{\mathrm{a}} \pm 0,00$ \\
\hline
\end{tabular}

Letras diferentes na mesma coluna diferem estatisticamente entre si ao nível de $5 \%$ pelo teste de Duncan. 
Tabela 2 - Degradação de ácido ascórbico em rações e premix expostos a ambiente climatizado $\left(20^{\circ} \mathrm{C} \pm 3^{\circ} \mathrm{C}\right)$.

\begin{tabular}{cccccc}
\hline $\begin{array}{c}\text { Período de } \\
\text { degradação \% (dias) }\end{array}$ & Amostra 1 & Amostra 2 & Amostra 3 & Amostra 4 & Amostra 5 \\
\hline T5 & $7,67^{\mathrm{f}} \pm 0,17$ & $13,32^{\mathrm{d}} \pm 0,88$ & $11,33^{\mathrm{f}} \pm 0,28$ & $0,72^{\mathrm{d}} \pm 0,23$ & $14,25^{\mathrm{f}} \pm 0,03$ \\
T10 & $37,33^{\mathrm{e}} \pm 0,05$ & $13,99^{\mathrm{d}} \pm 0,66$ & $12,66^{\mathrm{e}} \pm 0,00$ & $0,73^{\mathrm{d}} \pm 0,71$ & $21,25^{\mathrm{e}} \pm 0,00$ \\
T15 & $42,05^{\mathrm{d}} \pm 0,15$ & $15,99^{\mathrm{c}} \pm 0,55$ & $14,00^{\mathrm{d}} \pm 0,04$ & $2,22^{\mathrm{c}} \pm 0,08$ & $38,75^{\mathrm{d}} \pm 0,01$ \\
T20 & $43,33^{\mathrm{c}} \pm 0,28$ & $16,89^{\mathrm{bc}} \pm 0,71$ & $14,66^{\mathrm{c}} \pm 0,52$ & $3,71^{\mathrm{b}} \pm 0,21$ & $43,25^{\mathrm{c}} \pm 0,00$ \\
T25 & $45,69^{\mathrm{b}} \pm 0,08$ & $17,99^{\mathrm{b}} \pm 1,03$ & $16,07^{\mathrm{b}} \pm 0,09$ & $3,71^{\mathrm{b}} \pm 0,62$ & $46,00^{\mathrm{b}} \pm 0,00$ \\
T30 & $47,33^{\mathrm{a}} \pm 0,09$ & $19,32^{\mathrm{a}} \pm 0,98$ & $16,67^{\mathrm{a}} \pm 0,13$ & $5,18^{\mathrm{a}} \pm 0,19$ & $47,12^{\mathrm{a}} \pm 0,00$ \\
\hline
\end{tabular}

Letras diferentes na mesma coluna diferem estatisticamente entre si ao nível de $5 \%$ pelo teste de Duncan.

Tabela 3 - Degradação de ácido ascórbico em rações e premix submetidos a ambiente de estufa $(\%)\left(40^{\circ} \mathrm{C} \pm 3^{\circ} \mathrm{C}\right)$.

\begin{tabular}{cccccc}
\hline $\begin{array}{c}\text { Período de } \\
\text { degradação \% (dias) }\end{array}$ & Amostra 1 & Amostra 2 & Amostra 3 & Amostra 4 & Amostra 5 \\
\hline T5 & $18,00^{\mathrm{f}} \pm 0,09$ & $15,33^{\mathrm{f}} \pm 0,51$ & $13,33^{\mathrm{f}} \pm 0,32$ & $1,48^{\mathrm{f}} \pm 0,35$ & $38,75^{\mathrm{f}} \pm 0,00$ \\
T10 & $40,00^{\mathrm{e}} \pm 0,26$ & $18,66^{\mathrm{e}} \pm 0,50$ & $16,00^{\mathrm{e}} \pm 0,66$ & $2,96^{\mathrm{e}} \pm 0,65$ & $41,25^{\mathrm{e}} \pm 0,00$ \\
T15 & $43,33^{\mathrm{d}} \pm 0,22$ & $20,64^{\mathrm{d}} \pm 1,02$ & $18,66^{\mathrm{d}} \pm 0,76$ & $4,44^{\mathrm{d}} \pm 0,07$ & $45,25^{\mathrm{d}} \pm 0,00$ \\
T20 & $44,00^{\mathrm{c}} \pm 0,40$ & $24,67^{\mathrm{c}} \pm 0,10$ & $19,99^{\mathrm{c}} \pm 0,77$ & $6,67^{\mathrm{c}} \pm 0,54$ & $46,62^{\mathrm{c}} \pm 0,01$ \\
T25 & $46,66^{\mathrm{b}} \pm 0,23$ & $26,67^{\mathrm{b}} \pm 0,28$ & $21,33^{\mathrm{b}} \pm 0,44$ & $8,15^{\mathrm{b}} \pm 0,06$ & $51,63^{\mathrm{b}} \pm 0,01$ \\
T30 & $50,67^{\mathrm{a}} \pm 0,07$ & $29,90^{\mathrm{a}} \pm 0,80$ & $24,00^{\mathrm{a}} \pm 0,07$ & $10,36^{\mathrm{a}} \pm 0,64$ & $63,63^{\mathrm{a}} \pm 0,01$ \\
\hline
\end{tabular}

Letras diferentes na mesma coluna diferem estatisticamente entre si ao nível de $5 \%$ pelo teste de Duncan.

ascórbico e, após 30 dias, submetidos a ambiente de geladeira perderam 20,00 e 14,00\%, no ambiente climatizado, 19,32 e $16,67 \%$ e na estufa as perdas foram de 29,90 e $24,0 \%$, respectivamente. Observamos que as maiores perdas foram nas amostras submetida a estufa $\left(40^{\circ} \mathrm{C} \pm\right.$ $3^{\circ} \mathrm{C}$ ) e nas menores para as armazenadas na geladeira $\left(6^{\circ} \mathrm{C} \pm 2^{\circ} \mathrm{C}\right)$.

A amostra 04, ao ser analisada, inicialmente apresentou a menor quantidade de ácido ascórbico de $135 \mathrm{mg} / \mathrm{Kg}$. A degradação na mesma amostra analisada para um período de 30 dias de armazenamento na geladeira foi de $3,70 \%$, no ambiente climatizado foi de $5,18 \%$ e na estufa perdeu $10,36 \%$. Foram registradas perdas mais altas para a amostra 04 armazenada em estufa $\left(40^{\circ} \mathrm{C} \pm 3^{\circ} \mathrm{C}\right)$ e mais baixas para as armazenadas na geladeira $\left(6^{\circ} \mathrm{C} \pm 2^{\circ} \mathrm{C}\right)$, conforme as tabelas acima. Em comparação com as amostras analisadas, a amostra 04 foi a que menos perdeu ácido ascórbico nas diferentes temperaturas a que foi submetida.

As perdas de ácido ascórbico foram mais acentuadas na amostra 05 que corresponde ao premix vitamínico com maior quantidade inicial de ácido ascórbico (80.000mg/Kg). A degradação na mesma amostra analisada para um período de 30 dias de armazenamento na geladeira foi de $10,87 \%$, no ambiente climatizado foi de $47,12 \%$ e na estufa chegou a perder até $63,63 \%$. Foram registradas perdas mais altas para a amostra 05 armazenada em estufa $\left(40^{\circ} \mathrm{C} \pm 3^{\circ} \mathrm{C}\right)$ e mais baixas para a as armazenadas na geladeira $\left(6^{\circ} \mathrm{C} \pm 2^{\circ} \mathrm{C}\right)$. Em comparação com as outras amostras analisadas, a amostra 05 foi a que mais perdeu ácido ascórbico nas diferentes temperaturas a que foi submetida. O ácido ascórbico é alterado quando exposto por períodos prolongados a altas temperaturas, mostrandose que a quantidade excessiva desse ácido no premix favoreceu sua maior disponibilização à oxidação irreversível. Ficou caracterizado uma tendência à degradação de ácido ascórbico proporcional ao aumento da temperatura de armazenamento e concentração do mesmo na amostra. É importante observar que o ácido ascórbico é extremamente instável, reagindo com o oxigênio do ar, com a luz e até mesmo com a água que, tão logo exposto, sofre reações químicas que o destroem.

Os resultados obtidos evidenciaram que a degradação do ácido ascórbico foi afetada pelas condições e período de armazenamento. Sandnes \& Braekkan (1982) reportaram que rações para a aquicultura armazenadas a temperatura ambiente $\left(20^{\circ} \mathrm{C}\right)$ perderam quase todo o ácido ascórbico contido nestas rações depois de 16 semanas de armazenamento comparado a uma perda de $70 \%$ depois de 24 semanas de armazenamento a $4^{\circ} \mathrm{C}$. 
De acordo com as tabelas 4 e 5, para degradação na presença e ausência de luz, pode-se observar que a amostra 01 apresentou as maiores perdas de ácido ascórbico em relação as outras amostras, pois ela apresentou, inicialmente, $300 \mathrm{mg} / \mathrm{kg}$ e no $12^{\circ}$ dia submetida a um ambiente com presença de luz perdeu $58,33 \%$ e no escuro perdeu $43,33 \%$. A amostra 1 é a que possui característica física mais sutil, tendo o grão farináceo menor tamanho, proporcionando um maior ataque luminoso á ração.

As amostras 02 e 03 apresentaram comportamento semelhante apresentando no início $150 \mathrm{mg} / \mathrm{kg}$ e, após o $12^{\mathrm{a}}$ dia submetidas a presença de luz, sofreram perdas de $9,75 \%$ e $9,95 \%$, respectivamente, e na ausência de luz perdas de $9,66 \%$ e $9,99 \%$, respectivamente, demonstrando que o aumento do grão foi significativamente importante para maior proteção do ácido ascórbico, enquanto que a amostra 04 não houve perdas significativas de ácido por degradação. Podemos afirmar que como o Premix encontrava-se na forma de uma farinha de constituição fina e, portanto, sem proteção de alguma estrutura química, tornou-se mais suscetível ao ataque oxidativo. A amostra 05 que corresponde ao premix vitamínico, apresentou perda significativa, obtendo-se $80.000 \mathrm{mg} / \mathrm{kg}$ e no $12^{\circ}$ dia verificou-se uma perda de $26,50 \%$ no ambiente escuro e $33,00 \%$ com presença de luz.

Soliman et al. (1987) colocaram amostras de rações para camarões em caixas escuras e claras em temperatura ambiente $\left(22-24^{\circ} \mathrm{C}\right)$ durante $30,50,90$ e 120 dias e conseguiram os seguintes resultados para as caixas escuras: $82,86 \mathrm{mg} / \mathrm{Kg}$; $91,60 \mathrm{mg} / \mathrm{Kg} ; 0,00 \mathrm{mg} / \mathrm{Kg}$ e $0,00 \mathrm{mg} / \mathrm{Kg}$, respectivamente. Para as rações acondicionadas em caixas claras nas mesmas condições, os resultados foram: $86,56 \mathrm{mg} / \mathrm{Kg} ; 96,71 \mathrm{mg} / \mathrm{Kg}$; $0,00 \mathrm{mg} / \mathrm{Kg}$ e $0,00 \mathrm{mg} / \mathrm{Kg}$, respectivamente. As perdas do ácido ascórbico nas rações acondicionadas em caixas claras foram maiores que nas caixas escuras.

Avaliando a degradação do ácido ascórbico por lixiviação pode-se observar nas tabelas 6 e 7 que a amostra 01 apresentou as maiores perdas de ácido ascórbico em relação às outras amostras. Essa amostra apresentou inicialmente 300mg deste ácido / kg de ração e após 12 horas submetida a recipiente com água marinha e doce perdeu a mesma percentagem, 71,33\% nos dois tipos de água. As amostras 02 e 03, ao serem analisadas, inicialmente apresentaram $150 \mathrm{mg}$ de ácido ascórbico / kg de ração e após 12 horas submetidas a recipientes com água marinha e doce perderam as mesmas percentagens, $42,7 \%$.

Tabela 4 - Degradação na presença de luz do ácido ascórbico em rações e premix vitamínico.

\begin{tabular}{cccccc}
\hline $\begin{array}{c}\text { Período de } \\
\text { degradação } \\
\% \text { (dias) }\end{array}$ & Amostra 1 & Amostra 2 & Amostra 3 & Amostra 4 & Amostra 5 \\
\hline T4 & $40,00^{\mathrm{c}} \pm 0,05$ & $9,87^{\mathrm{a}} \pm 0,22$ & $10,00^{\mathrm{a}} \pm 0,55$ & $0,00^{\mathrm{b}} \pm 0,00$ & $15,00^{\mathrm{c}} \pm 0,00$ \\
T8 & $55,00^{\mathrm{b}} \pm 0,08$ & $9,99^{\mathrm{a}} \pm 0,82$ & $9,95^{\mathrm{a}} \pm 0,60$ & $0,18^{\mathrm{ab}} \pm 0,15$ & $28,00^{\mathrm{b}} \pm 0,00$ \\
T12 & $58,33^{\mathrm{a}} \pm 0,02$ & $9,75^{\mathrm{a}} \pm 0,43$ & $9,95^{\mathrm{a}} \pm 0,60$ & $0,33^{\mathrm{a}} \pm 0,28$ & $33,00^{\mathrm{a}} \pm 0,00$ \\
\hline
\end{tabular}

Letras diferentes na mesma coluna diferem estatisticamente entre si ao nível de $5 \%$ pelo teste de Duncan.

Tabela 5 - Degradação na ausência de luz do ácido ascórbico em rações e premix vitamínico.

\begin{tabular}{cccccc}
\hline $\begin{array}{c}\text { Período de } \\
\text { degradação } \% \\
\text { (dias) }\end{array}$ & Amostra 1 & Amostra 2 & Amostra 3 & Amostra 4 & Amostra 5 \\
\hline T4 & $36,56^{\mathrm{c}} \pm 0,23$ & $9,99^{\mathrm{a}} \pm 0,89$ & $9,74^{\mathrm{a}} \pm 0,45$ & $0,03^{\mathrm{b}} \pm 0,02$ & $10,00^{\mathrm{c}} \pm 0,00$ \\
T8 & $40,00^{\mathrm{b}} \pm 0,04$ & $9,99^{\mathrm{a}} \pm 0,67$ & $10,00^{\mathrm{a}} \pm 0,41$ & $0,33^{\mathrm{a}} \pm 0,29$ & $20,95^{\mathrm{b}} \pm 0,00$ \\
T12 & $43,33^{\mathrm{a}} \pm 0,13$ & $9,66^{\mathrm{a}} \pm 0,67$ & $9,99^{\mathrm{a}} \pm 0,88$ & $0,01^{\mathrm{b}} \pm 0,00$ & $26,50^{\mathrm{a}} \pm 0,00$ \\
\hline
\end{tabular}

Letras diferentes na mesma coluna diferem estatisticamente entre si ao nível de 5\% pelo teste de Duncan.

Tabela 6 - Degradação de ácido ascórbico por lixiviação em ambiente marinho.

\begin{tabular}{ccccc}
\hline $\begin{array}{c}\text { Período de } \\
\text { degradação (horas) }\end{array}$ & Amostra 1 & Amostra 2 & Amostra 3 & Amostra 4 \\
\hline T0 & $0,00^{\mathrm{d}} \pm 0,00$ & $0,00^{\mathrm{d}} \pm 0,00$ & $0,00^{\mathrm{d}} \pm 0,00$ & $0,00^{\mathrm{d}} \pm 0,00$ \\
T3 & $22,33^{\mathrm{c}} \pm 0,15$ & $13,99^{\mathrm{c}} \pm 1,06$ & $17,33^{\mathrm{c}} \pm 0,24$ & $11,85^{\mathrm{c}} \pm 0,55$ \\
T6 & $57,00^{\mathrm{b}} \pm 0,08$ & $39,33^{\mathrm{b}} \pm 0,70$ & $37,99^{\mathrm{b}} \pm 0,51$ & $31,11^{\mathrm{b}} \pm 0,06$ \\
T12 & $71,33^{\mathrm{a}} \pm 0,09$ & $42,67^{\mathrm{a}} \pm 0,20$ & $42,66^{\mathrm{a}} \pm 0,51$ & $37,04^{\mathrm{a}} \pm 0,45$ \\
\hline
\end{tabular}

Letras diferentes na mesma coluna diferem estatisticamente entre si ao nível de 5\% pelo teste de Duncan. 
A amostra 04, ao ser analisada, inicialmente apresentou a menor quantidade de ácido ascórbico, 135 $\mathrm{mg} / \mathrm{kg}$ e quando comparada com as outras amostras analisadas foi a que menos perdeu ácido ascórbico quando submetida às águas dos diferentes ambientes. Na água marinha a perda foi de $37,04 \%$ e na água doce foi de 40,0\%, portanto, a perda maior foi na água doce. Ficou demonstrado, primeiramente, que o teor de sais não foi fator de alteração da estabilidade do ácido ascórbico nas rações, tendo em vista as semelhanças nos teores em água estuariana e doce. Também mostrou-se um comportamento diferenciado em relação ao fato de que o Premix vitamínico teve menor degradação em relação às rações em estudo, mostrando que a composição do mesmo de alguma forma favoreceu sua estabilidade. Evidenciando o fato de que a ração possui um teor considerável de lipídios que favorece o fenômeno oxidativo, principalmente em ambiente rico em oxigênio.

Tabela 7 - Degradação de ácido ascórbico por lixiviação em ambiente estuarino.

\begin{tabular}{ccccc}
\hline $\begin{array}{c}\text { Período de } \\
\text { degradação } \\
\text { (horas) }\end{array}$ & Amostra 1 & Amostra 2 & Amostra 3 & Amostra 4 \\
\hline T0 & $0,00^{\mathrm{d}} \pm$ & $0,00^{\mathrm{d}} \pm$ & $0,00^{\mathrm{d}} \pm$ & $0,00^{\mathrm{d}} \pm$ \\
& 0,00 & 0,00 & 0,00 & 0,00 \\
T3 & $28,00 \pm$ & $22,66^{\mathrm{c}} \pm$ & $18,66^{\mathrm{c}} \pm$ & $16,30^{\mathrm{c}} \pm$ \\
& 0,19 & 0,96 & 0,55 & 0,71 \\
$\mathrm{~T} 6$ & $62,00^{\mathrm{b}} \pm$ & $38,66^{\mathrm{b}} \pm$ & $39,33^{\mathrm{b}} \pm$ & $34,07^{\mathrm{b}} \pm$ \\
& 0,11 & 0,74 & 0,61 & 0,10 \\
$\mathrm{~T} 12$ & $71,33^{\mathrm{a}} \pm$ & $42,67^{\mathrm{a}} \pm$ & $42,67^{\mathrm{a}} \pm$ & $40,00^{\mathrm{a}} \pm$ \\
& 0,18 & 0,31 & 0,02 & 0,36 \\
\hline
\end{tabular}

Letras diferentes na mesma coluna diferem estatisticamente entre si ao nível de $5 \%$ pelo teste de Duncan.

Solimam et al. (1987) realizaram um estudo onde avaliaram as perdas de ácido ascórbico contido em rações para aquicultura. As rações foram imersas em água por períodos de tempo que variaram em cada temperatura da água ( 20 e $28^{\circ} \mathrm{C}$ ). Os resultados obtidos nesse experimento foram os seguintes: ocorreram perdas de $36,48 \%$ para as rações imersas em água com temperatura de $20^{\circ} \mathrm{C}$ e $52,50 \%$ para as rações imersas em água com temperatura de $28^{\circ} \mathrm{C} . \mathrm{O}$ tempo crescente da imersão e o aumento da temperatura da água conduziram a uma alta lixiviação.

\section{CONCLUSÕES}

Evidenciou-se que o teor de ácido ascórbico foi afetado pelas condições e períodos de armazenamento, quando submetidas aos níveis de temperatura (geladeira, ambiente climatizado e estufa), sofrendo diminuição na quantidade de ácido ascórbico em função do tempo e da temperatura, sendo que as perdas maiores ocorreram em ambiente de estufa.

A ração comercial (amostra 01) e o premix vitamínico (amostra 05) foram as que perderam maior quantidade de ácido ascórbico, quando expostas à luz por um período de até 12 dias. A concentração de ácido ascórbico em rações sofre interferência da incidência de luz, ou seja, degradação fotoquímica, sendo necessário, portanto, cuidados com o armazenamento destas rações, dando prioridade a ambientes com baixa luminosidade.

Tendo-se em vista que o ácido ascórbico é hidrossolúvel, a lixiviação torna-se um fator limitante para o uso de rações na aquicultura. No presente estudo, ocorreu uma correlação entre a degradação do ácido e o tempo de imersão, sendo necessário o uso de rações melhoradas com a utilização de alimentadores automáticos e/ou o uso de rações palatáveis, que serão rapidamente consumidas, como também a utilização de formas protegidas de ácido ascórbico para diminuir a lixiviação.

\section{REFERÊNCIAS BIBLIOGRÁFICAS}

\author{
ALMEIDA, S.A.; CESAR, J.R.O.; BEZERRA, F.J.S.; \\ CARVALHO, M.C. Estudo preliminar do cultivo de \\ Litopenaeus vannamei (Bonne, 1931) em tanques com \\ diferentes densidades de estocagem. In: CONLAEP, 11., \\ 2004, Piracicaba. Anais... Piracicaba: ESALQ, 2004. v.2, \\ p.648-653.
}

\section{ASSOCIATION OF OFFICIALANALYTICAL CHEMISTS. Official methods of analysis. 27.ed. Washington, DC, 1984. 845p.}

BENASSI, M.T.; ANTUNES, J.A. Comparison of metaphosphoric and acids as extraction solutions for the detremination of vitamin in selected vegetable. Arquivo de Biologia e Tecnologia, São Paulo, v.31, p.507-513, 1988.

EVA, J.K.; FIFILED, R.; RICKETT, M. Decomposition of supplementary vitamin $\mathrm{C}$ in diets compounded for laboratory animals. Laboratório Animal, São Paulo, v.10, p.157-159, 1976.

FISH FARMING INTERNATIONAL. Vitamin C has high place in feeds for farm fish. Fish, New York, v.11, n.6, p.18-24, 1984. 
KUBITZA, F. Nutrição e alimentação dos peixes cultivados. 3.ed. rev. e ampl. Jundiaí: F.Kubitza, 1999. 123p.

O'KEEFE, T. Ascorbic acid and stable ascorbate esters as sources of vitamin $\mathbf{C}$ in aquaculture feeds. Singapore: American Soybean Association-United Soybean Board, 2001. 8p. (ASA Technical Bulletin, AQ48-2001).

Disponível em: <http://www.asasea.com/technical/aq48.' 2001.html>. Acesso em: 17 abr. 2006.

ORMOND, J.P.; TÁBUAS, A.G.; PIRES, P.R. A carcinicultura brasileira. BNDES Setorial, Rio de Janeiro, n.19, p.19-118, mar. 2004.
SANDNES, K.; BRAEKKAN, O.R. Ascorbic acid and the reproductive cycle of ovaries in cod (Gadus morrhua). Comparative Biochemistry and Physiology, New York, v.70, p.545-546, 1982.

SOLIMAN, A.K.; JAUNCEY, K.; ROBERTS, R.J. Stability of L-Ascorbic acid (Vitamin C) and its forms in fish feeds during processing, storage and leaching. Aquaculture, Amsterdam, v.60, n.2, p.73-83, 1987.

TACON, A.G.J. Vitamin nutrition in shrimp and fish. In: AQUACULTURE FEED PROCESSING AND NUTRITION WORKSHOP, 1991, Singapore. Procedings... Singapore: American Soybean Association, 1991 